\title{
Outcomes of Endovascular Intervention for Salvage of Failing Hemodialysis Access
}

\author{
Terence LX Tan, BMedSci, Kyin K May, MBBS, Peter A Robless, MD, MBChB, and Pei Ho, MBBS, FRCS, \\ FHKAM
}

Objective: To investigate the effectiveness of endovascular balloon angioplasty to preserve the patency of failing hemodialysis arteriovenous fistulas (AVF) and prosthetic arteriovenous grafts (AVG).

Methods: Patients on hemodialysis who received endovascular intervention for access problems were retrospectively analyzed. Fistulography was performed on patients who were suspected to have access stenosis and balloon angioplasty performed in the same setting if a stenosis of $\geq \mathbf{5 0 \%}$ is detected. Patients were followed up for post-operative complications and access restenosis or failure.

Results: 42 hemodialysis patients with 44 access sites (29 AVFs, 15 AVGs) required endovascular balloon angioplasty. There were no perioperative complications. Technical success rate was $100 \%$. Median time from initial access creation to first balloon angioplasty was 13 months (2-146 months) for AVFs and 8 months (2-71 months) for AVGs. 19 of 44 patients subsequently developed restenosis. Median time for restenosis or access failure was 11 months (1-18 months) for AVFs and 5 months (1-10 months) for AVGs. Kaplan-Meier analysis for access patency after endovascular intervention showed $72 \%$ patency at 6 months and $32 \%$ at 12 months.

Conclusions: Endovascular balloon angioplasty is effective in restoring patency of failing hemodialysis accesses. Recurrence is common, and repeat interventions are required.

Key words: angioplasty, arteriovenous fistula, arteriovenous graft, renal failure, hemodialysis

\section{INTRODUCTION}

A utogenous arteriovenous fistulas (AVF) and prosthetic arteriovenous grafts (AVG) are necessary for chronic end-stage renal failure patients on hemodialysis. AVFs are the preferred initial hemodialysis access due to their longer patency than AVGs.) AVGs, however, remain

Department of Cardiac, Thoracic and Vascular Surgery, National University Heart Centre, National University Hospital, Singapore

Received: November 7, 2010; Accepted: March 9, 2011 Corresponding author: Pei Ho, MBBS, FRCS, FHKAM. Department of Cardiac, Thoracic and Vascular Surgery, National University Heart Centre, National University Hospital, Singapore, 9/F NUHS Tower Block, 1E Kent Ridge Road, 119228, Singapore

Tel: +65-6772-2859, Fax: +65-6776-6475

E-mail: surhp@nus.edu.sg clinically important in patients whom AVFs are not feasible, and possibly in special populations such as the elderly. $^{2-4)}$

Hemodialysis accesses are prone to failure due to thrombosis, usually concomitant with stenosis over the anastomosis or outflow vein. Access thrombosis frequently requires semi-emergent salvage intervention, but outcomes are generally unfavorable. Patients eventually may require multiple salvage procedures to restore functionality or creation of a new access. Some patients require placement of central venous catheters in the interim whilst the hemodialysis access becomes fully functional. Alternatively, to prevent access failure, clinicians can monitor the performance of these accesses and prophylactically provide interventions to rectify the hemodynamic problems and prolong their patency. ${ }^{5}$

Our service currently provides endovascular interventions to haemodialysis patients with failing access. This 
Table 1 Patient demographics

\begin{tabular}{lc}
\hline & Total \\
\hline Age (Mean \pm SD) & $59.8 \pm 11.3$ \\
Sex: Male $(\%)$ & $24(57.1 \%)$ \\
Sex: Female $(\%)$ & $18(42.9 \%)$ \\
Diabetes mellitus & $32(73 \%)$ \\
Hypertension & $37(84 \%)$ \\
Ischaemic heart disease & $18(41 \%)$ \\
Hyperlipidaemia & $25(57 \%)$ \\
Peripheral vascular disease & $9(21 \%)$ \\
Stroke & $6(14 \%)$ \\
Smokers, active & $6(14 \%)$ \\
\hline
\end{tabular}

study aims to review the effectiveness of endovascular intervention for preservation of failing accesses.

\section{Patients ANd Methods}

This study is approved by the institutional review board at our hospital. From August 2008 to March 2010, hemodialysis patients who received endovascular treatment for their dialysis access problem in the Vascular Surgery division of National University Hospital, Singapore, were retrospectively analyzed. The feature of failing AVFs or AVGs included one or more of the following: 1) Reduced thrill of vascular access assessed by clinical palpation by a vascular surgeon (P Ho), 2) Documented decreased dialysis flow rate defined by KDOQI guidelines $^{6}$ (access flow less than $600 \mathrm{ml} / \mathrm{min}$, or less than $1000 \mathrm{ml} / \mathrm{min}$ with a more than $25 \%$ decrease over a four month period, 3) Documented increased venous pressure during dialysis as defined by KDOQI guidelines $^{6}$ (venous pressure of more than $150 \mathrm{mmHg}$ or a trend of persistent increasing pressure over time) 4) Stenosis detected by duplex ultrasound, 5) Non-maturation of an AVF six weeks post-creation. Patient demographics and co-morbidities were documented.

Fistulograms were offered to all patients with one or more of the above-mentioned features of vascular access failure. The degree of stenosis in the peri-anastomotic region, fistula/graft, outflow vein and central veins were assessed during fistulography. Endovascular intervention in the form of balloon angioplasty was performed in the same setting if a stenosis of $50 \%$ or more of the vessel diameter is detected on angiography, using either a simple Wanda balloon (Boston Scientific, Natick, MA, USA) or a Peripheral cutting balloon (Boston Scientific, Natick, MA, USA). Cutting balloons were utilized for high grade
Table 2 Frequency of indication for fistulogram

\begin{tabular}{lc} 
Evidence of failing dialysis access & $\begin{array}{c}\text { Number of cases } \\
(\%)\end{array}$ \\
\hline Decreased dialysis flow rates & $26(59.1 \%)$ \\
Stenosis on duplex ultrasound & $7(15.9 \%)$ \\
Lack of fistula maturation & $4(9.1 \%)$ \\
Increased venous pressures & $2(4.5 \%)$ \\
Suspected thrombosis & $2(4.5 \%)$ \\
Difficulty in dialysis cannulation & $2(4.6 \%)$ \\
Poor thrill on clinical examination & $1(2.3 \%)$ \\
\hline
\end{tabular}

stenosis ( $\geq 90 \%$ stenosis) or total occlusion on fistulography, or when a simple balloon did not result in satisfactory restoration of access patency (re-stenosis $>50 \%$ ). Completion fistulogram was performed immediately after angioplasty to determine success of the procedure. All patients were observed in the day surgery ward for two to three hours and discharged if no acute complications (bleeding or thrombosis) were detected. AVFs and AVGs, which were functional for dialysis pre-intervention, were used for dialysis the day after angioplasty.

Data are given as percentages and medians (range). Patency rates of hemodialysis access after initial salvage angioplasty were calculated according to the KaplanMeier method. Statistical analyses were performed using PASW Statistics 18 (IBM SPSS, Chicago, IL, USA). The fistulograms of the vascular accesses that require secondary intervention were reviewed to compare the sites of obstruction in the initial and subsequent intervention.

\section{RESULTS}

During the study period, 42 patients with a total of 44 hemodialysis accesses had stenosis confirmed by fistulogram and subsequently underwent balloon angioplasty for rectification of hemodynamic obstruction. 24 (57.1\%) patients were male and 18 (42.9\%) were female, with a mean age of $59.8 \pm 11.3$ (Range, 28-82). Medical co-morbidities were common among the studied population: 32 patients had diabetes mellitus (73\%), 37 had hypertension (84\%), 18 had ischaemic heart disease (41\%), 25 had hyperlipidemia (57\%), 9 had peripheral vascular disease (21\%), and 6 had a history of stroke (14\%). 6 of the patients were active smokers (14\%) (Table 1).

Of the 44 hemodialysis accesses, 29 (65.9\%) were AVFs, and 15 (34.1\%) were AVGs. Frequencies of the 
Table 3 Median time from access creation to first endovascular intervention

\begin{tabular}{lcc}
\hline & Median (months) & Range (min-max) \\
\hline AVF $(\mathrm{n}=29)$ & 13 & $2-146$ \\
AVG $(\mathrm{n}=15)$ & 8 & $2-71$ \\
Total $(\mathrm{n}=44)$ & 11 & $2-146$ \\
\hline
\end{tabular}

Table 4 Median time from first endovascular intervention to subsequent access failure

\begin{tabular}{lcc}
\hline & Median (months) & Range (min-max) \\
\hline AVF $(\mathrm{n}=10)$ & 11 & $1-18$ \\
$\operatorname{AVG}(\mathrm{n}=9)$ & 5 & $1-10$ \\
Total $(\mathrm{n}=19)$ & 8 & $1-18$ \\
\hline
\end{tabular}

chief indication for fistulogram in the 44 accesses studied are listed in Table 2. As shown, the majority of patients were noted to have decreased access flow rates as measured during dialysis. The median time from access creation to the time of intervention was 13 months (Range, 2-146 months) for AVFs, and 8 months (Range, 2-71 months) for AVGs, yielding an average, median interval of 11 months (Range, 2-146) for all 44 accesses (Table 3).

The procedure success rate was $100 \%$. No complications, notably bleeding and thrombosis, were detected after the interventions. Of the four non-mature AVFs, three eventually matured after angioplasty and was able to be used for dialysis. The remaining one AVF failed to mature, and this patient required the creation of a new vascular access. All other salvaged accesses were functional for dialysis immediately post-intervention.

After initial angioplasty, patients were followed up for a median of 5 months (Range, 1-18 months). 19 of the 44 (43.2\%, 10 AVFs and 9 AVGs) accesses initially revised eventually failed again. There was no significant difference between the proportion of AVFs and AVGs that required a secondary procedure $(34.5 \%$ AVFs, $60.0 \%$ AVGs, $\mathrm{p}=0.097)$. On these 19 accesses, 11 accesses (57.9\%) required a second angioplasty to restore patency, 5 (26.3\%) thrombosed, and required the creation of new hemodialysis access, 1 (5.3\%) required a jump graft, 1 (5.3\%) required surgical revision, and 1 (5.3\%) was converted to peritoneal dialysis after the access thrombosed.

Analysis of fistulographs of the 11 accesses that required a second angioplasty showed that stenosis recurred at the same site in $7(63.6 \%)$ accesses, occurred in both the old and a new site in $2(18.2 \%)$ accesses, extended contiguously in $1(9.1 \%)$ access, and receded from two sites to one in $1(9.1 \%)$ access.

The median time from initial salvage angioplasty to re-intervention was 11 months (Range, 1-18 months) for AVFs and 5 months (Range, 1-10 months) for AVGs, yielding an average, median interval of 8 months (Range, 1-18 months) (Table 4). During the study period, four patients died from causes unrelated to hemodialysis access procedure (two from acute myocardial infarction, one from septic shock and one from a bleeding duodenal ulcer). Kaplan-Meier curves for hemodialysis accesses after initial balloon angioplasty showed a patency rate of $72 \%$ at 6 months and $32 \%$ at 12 months (Fig. 1).

\section{DiscuSSION}

Hemodialysis accesses in the form of AVFs or AVGs are integral to sustaining life in patients with established end-stage kidney disease. However, AVFs, and especially AVGs, are prone to stenosis and thrombosis which may preclude hemodialysis. Abandonment of a primary access frequently necessitates the creation of secondary and even tertiary accesses. The need for additional interventions $^{7)}$ in a group of patients already immunocompromised by renal disease results in increased hospital stays, increased economic costs, ${ }^{8}$ and increased morbidity and mortality.

Thus, it is important for nephrologists and vascular surgeons to adopt strategies that will minimize morbidity and maximize quality of life when dealing with patients on hemodialysis. In 2006, the Dialysis Outcomes Quality Initiative (DOQI) published by the US National Kidney Foundation1 strongly recommended the placement of AVFs as a primary access for hemodialysis. This was based on research showing that patients with AVFs had a 


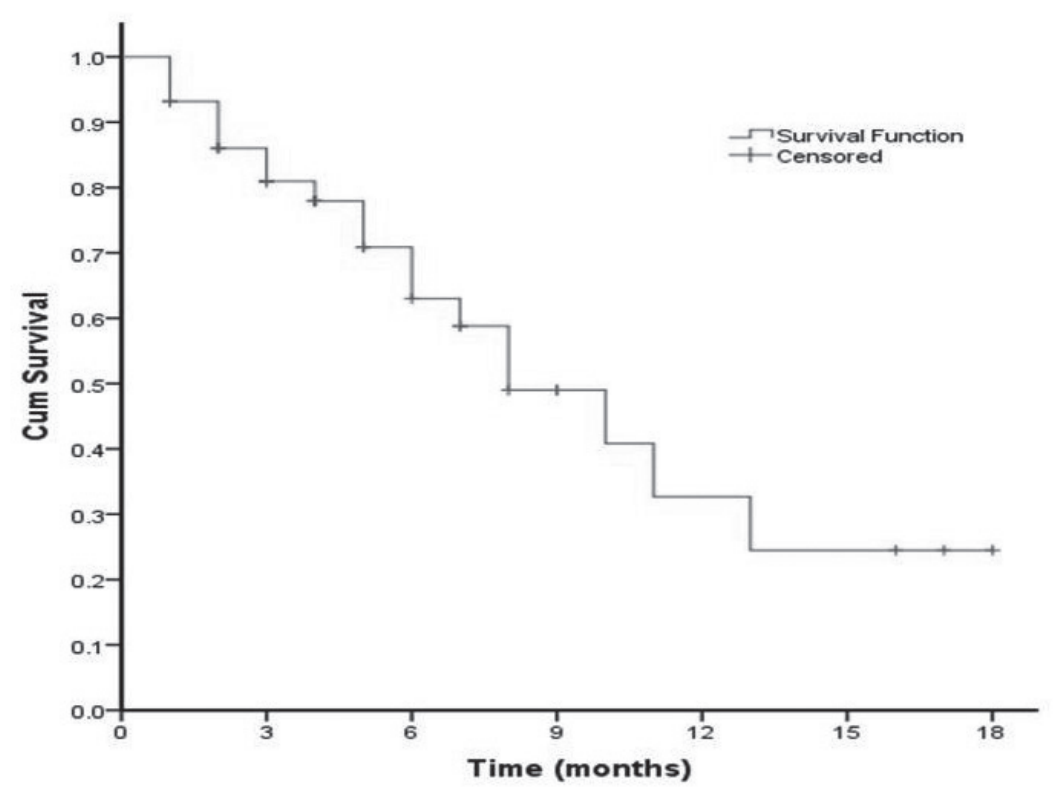

Fig. 1 Kaplan-Meier analysis for dialysis access patency after first endovascular intervention.

better overall quality of life than those with AVGs.. Indeed, most studies have subsequently showed that AVFs are beneficial compared to AVGs, with AVFs having greater primary patencies compared to AVGs. ${ }^{10-12)}$ This finding correlates with the results of the present study, where the median time needed from access creation to initial intervention was 13 months for AVFs, and 8 months for AVGs. Moreover, after the initial intervention, the time to develop restenosis or thrombosis was shorter in AVGs compared to AVFs. The shorter time taken for AVGs to develop stenosis and subsequent restenosis post-intervention indicates that patients with AVGs require more frequent interventions to maintain graft patency. AVGs, however, are nonetheless required in patients with inadequate upper arm veins and in those who have exhausted their autogenous venous capital. Some studies have shown that elderly ${ }^{3)}$ and diabetic patients ${ }^{2)}$ with AVGs have better access patency rates when compared to similar patients with AVFs, indicating that patient co-morbidities and demographics may play an important role in determining the best hemodialysis access type.

In this study, decreased flow rates and increased venous pressures measured during dialysis were the chief reasons suggesting that a patient's hemodialysis access was failing. This suggests that routine monitoring of access performance during dialysis is useful in picking up early access failure for salvage intervention. Some cli- nicians advocate a regular surveillance program involving dialysis flow parameters ${ }^{1,13)}$ or duplex ultrasound imaging, ${ }^{14)}$ in addition to standard of care clinical monitoring to detect and prophylactically intervene with failing hemodialysis accesses. Research has shown that regular surveillance may lead to improved quality of life for dialysis patients, ${ }^{15)}$ possibly from a reduction in the quantity of time spent in hospitals due to complications of access failure. ${ }^{16)}$ This benefit, however, has to be viewed in the context of unnecessarily exposing patients to increased numbers of salvage interventions as a consequence of routine surveillance. ${ }^{17}{ }^{18)}$ Importantly, there is substantial evidence that regular surveillance of AVGs may not decrease the risk of graft thrombosis, ${ }^{19)}$ nor prolong the time to graft abandonement. ${ }^{18,20)}$ On the other hand, regular surveillance of AVFs does decrease the risk of access thrombosis, ${ }^{21)}$ but its effect on improving AVF patency is uncertain, with clinical trials producing conflicting results. ${ }^{21,22)}$ Surveillance protocols using ultrasonography in previous studies have mostly monitored accesses every 3-4 monthly. ${ }^{18,23,24)}$ Given that the median time to initial and secondary intervention in this study for AVFs is 13 months and 11 months respectively, it is likely that a six-month surveillance programme will detect significant stenosis amenable to prophylactic intervention. A prospective comparative study investigating the patency rates of hemodynamic accesses and cost effectiveness under different surveillance intervals will 
be needed to determine what the optimal surveillance interval should be. This longer interval between surveillance points may have the advantages of decreasing healthcare resources and costs required, also prevent patients from undergoing multiple unnecessarily procedures.

Percutaneous transluminal angioplasty (PTA) is the mainstay of treatment in stenosed hemodialysis access. With current techniques, PTA is a safe and useful intervention to restore access patency and preserve venous capital for future AVF or AVG creation. ${ }^{25)}$ PTA restores the luminal diameter of venous fistula by stretching and dissection of the vessel wall. This induces vascular damage and may cause subsequent restenosis. ${ }^{26)}$ Whether the mechanism of venous restenosis is similar to arterial restenosis is uncertain. However, in our experience, venous restenosis seems to recur more frequently than that of its arterial counterpart. Cutting balloon angioplasty reduces the amount of arterial wall damage by inducing a controlled fracture of atherosclerotic plaque. It is currently uncertain if cutting balloon angioplasty will reduce the recurrence rate of venous stenosis as compared to conventional balloon angioplasty. A recent study by Kariya et al. $^{27)}$ showed no significant differences between these two techniques. Stenting of access stenosis is usually regarded as a second line option to balloon angioplasty and is usually performed only if balloon angioplasty is unsuccessful or with dissection. However, there is some evidence to suggest that stenting alone can improve patency outcomes in both AVFs and AVGs. ${ }^{28)} \mathrm{A}$ recent study ${ }^{29,30)}$ has also shown that combined balloon angioplasty and stent-grafting confers an improved patency rate in AVGs with venous anastomotic stenosis over balloon angioplasty alone. It is not known if similar findings extend to AVFs.

\section{Conclusion}

Endovascular intervention in the form of balloon angioplasty is a safe and effective treatment for failing AVFs and AVGs. Re-stenosis is common and often requires further intervention in the form of repeat angioplasty.

\section{REFERENCES}

1) Gilmore J. KDOQI clinical practice guidelines and clinical practice recommendations-2006 updates. Nephrol Nurs J 2006; 33: 487-8.
2) Woods JD, Turenne MN, Strawderman RL, Young EW, Hirth RA, Port FK, et al. Vascular access survival among incident haemodialysis patients in the United States. Am J Kidney Dis 1997; 30: 50-7.

3) Chan MR, Sanchez RJ, Young HN, Yevzlin AS. Vascular access outcomes in the elderly haemodialysis population: A USRDS study. Semin Dial 2007; 20: 606-10.

4) Richardson AI 2nd, Leake A, Schmieder GC, Biuckians A, Stokes GK, Panneton JM, et al. Should fistulas really be first in the elderly patient? J Vasc Access 2009; 10: 199-202.

5) Turmel-Rodrigues L, Mouton A, Birmele B, Billaux L, Ammar N, Grézard O, et al. Salvage of immature forearm fistulas for haemodialysis by interventional radiology. Nephrol Dial Transplant 2001; 16: 2365-71.

6) Foundation NK. KDOQI clinical practice guidelines and clinical practice recommendations for 2006 updates: haemodialysis adequacy, peritoneal dialysis adequacy and vascular access. Am J Kidney Dis 2006; 48 (suppl 1): S1-S322.

7) Hingorani A, Ascher E, Kallakuri S, Greenberg S, Khanimov Y. Impact of reintervention for failing upper-extremity arteriovenous autogenous access for haemodialysis. J Vasc Surg 2001; 34: 1004-9.

8) Bittl JA, Cohen DJ, Seek MM, Feldman RL. Economic analysis of angiography and preemptive angioplasty to prevent haemodialysis-access thrombosis. Catheter Cardiovasc Interv 2009; 75: 14-21.

9) Bazan HA. Why "fistula first" matters: increased durability, less interventions, and decreased costs. Catheter Cardiovasc Interv 2010; 75: 22.

10) Hakim R, Himmelfarb J. Haemodialysis access failure: a call to action. Kidney Int 1998; 54: 1029-40.

11) Coburn MC, Carney WI Jr. Comparison of basilic vein and polytetrafluoroethylene for brachial arteriovenous fistula. J Vasc Surg 1994; 20: 896-902.

12) Matsuura JH, Rosenthal D, Clark M, Shuler FW, Kirby L, Shotwell M, et al. Transposed basilic vein versus polytetrafluorethylene for brachial-axillary arteriovenous fistulas. Am J Surg 1998; 176: 219-1.

13) Gallego Beuter JJ, Hernandez Lezana A, Herrero Calvo J, Moreno Carriles R. Early detection and treatment of haemodialysis access dysfunction. Cardiovasc Intervent Radiol 2000; 23: 40-6.

14) Sands J, Young S, Miranda C. The effect of Doppler flow screening studies and elective revisions on dialysis access failure. ASAIO J 1992; 38: M524-527.

15) Henry ML. Routine surveillance in vascular access for haemodialysis. Eur J Vasc Endovasc Surg 2006; 32: $545-8$.

16) Dossabhoy NR, Ram SJ, Nassar R, Work J, Eason JM, Paulson WD. Stenosis surveillance of haemodialysis grafts by duplex ultrasound reduces hospitalizations and cost of care. Semin Dial 2005; 18: 550-7.

17) Wijnen E, Planken N, Keuter X, Kooman JP, Tordoir $\mathrm{JH}$, de Haan MW, et al. Impact of a quality improvement programme based on vascular access flow moni- 
toring on costs, access occlusion and access failure. Nephrol Dial Transplant 2006; 21: 3514-9.

18) Robbin ML, Oser RF, Lee JY, Heudebert GR, Mennemeyer ST, Allon M. Randomized comparison of ultrasound surveillance and clinical monitoring on arteriovenous graft outcomes. Kidney Int 2006; 69: 730-5.

19) Allon M, Robbin ML. Haemodialysis vascular access monitoring: current concepts. Haemodial Int 2009; 13: 153-62.

20) Dember LM, Holmberg EF, Kaufman JS. Randomized controlled trial of prophylactic repair of haemodialysis arteriovenous graft stenosis. Kidney Int 2004; 66: 390-8.

21) Tonelli M, James M, Wiebe N, Jindal K, Hemmelgarn B. Ultrasound monitoring to detect access stenosis in haemodialysis patients: a systematic review. Am J Kidney Dis 2008; 51: 630-40.

22) Tessitore N, Lipari G, Poli A, Bedogna V, Baggio E, Loschiavo C, et al. Can blood flow surveillance and pre-emptive repair of subclinical stenosis prolong the useful life of arteriovenous fistulae? A randomized controlled study. Nephrol Dial Transplant 2004; 19: 2325-33.

23) Scaffaro LA, Bettio JA, Cavazzola SA, Campos BT, Burmeister JE, Pereira RM, et al. Maintenance of haemodialysis arteriovenous fistulas by an interventional strategy: clinical and duplex ultrasonographic surveillance followed by transluminal angioplasty. J Ultrasound Med 2009; 28: 1159-65.
24) Malik J, Tuka V, Chytilova E, Mokrejsova M, Masek M, Slavikova M. Low-flow polytetrafluoroethylene accesses: ultrasound surveillance and preemptive interventions ensure long-term patency. Kidney Blood Press Res 23 2010; 33: 181-5.

25) Beathard GA. Fistula salvage by endovascular therapy. Adv Chronic Kidney Dis 2009; 16: 339-51.

26) Davidson CJ, Newman GE, Sheikh KH, Kisslo K, Stack RS, Schwab SJ. Mechanisms of angioplasty in haemodialysis fistula stenoses evaluated by intravascular ultrasound. Kidney Int 1991; 40: 91-5.

27) Kariya S, Tanigawa N, Kojima H, Komemushi A, Shomura Y, Shiraishi T, et al. Primary patency with cutting and conventional balloon angioplasty for different types of haemodialysis access stenosis. Radiology 2007; 243: 578-87.

28) Chan MR, Bedi S, Sanchez RJ, Young HN, Becker YT, Kellerman PS, et al. Stent placement versus angioplasty improves patency of arteriovenous grafts and blood flow of arteriovenous fistulae. Clin J Am Soc Nephrol 2008; 3: 699-705.

29) Dolmatch BL. Stent graft versus balloon angioplasty for failing dialysis access grafts: A long-awaited advance in the treatment of permanent haemodialysis access. J Vasc Access 2010; 11: 89-91.

30) Haskal ZJ, Trerotola S, Dolmatch B, Schuman E, Altman S, Mietling S, et al. Stent graft versus balloon angioplasty for failing dialysis-access grafts. N Engl J Med 2010; 362: 494-503. 